\title{
Quantum-Gravitational Effects on Primordial Power Spectra in Slow-Roll Inflationary Models
}

\author{
David Brizuela ${ }^{1}$ and Manuel Krämer ${ }^{2, *}$ \\ 1 Fisika Teorikoa eta Zientziaren Historia Saila, UPV/EHU, 644 P.K., 48080 Bilbao, Spain; \\ david.brizuela@ehu.eus \\ 2 Instytut Fizyki, Uniwersytet Szczeciński, Wielkopolska 15, 70-451 Szczecin, Poland \\ * Correspondence: manuel.kraemer@usz.edu.pl
}

Received: 30 November 2017; Accepted: 3 January 2018; Published: 8 January 2018

\begin{abstract}
We review the computation of the power spectra of inflationary gauge-invariant perturbations in the context of canonical quantum gravity for generic slow-roll models. A semiclassical approximation, based on an expansion in inverse powers of the Planck mass, is applied to the complete Wheeler-DeWitt equation describing a perturbed inflationary universe. This expansion leads to a hierarchy of equations at consecutive orders of the approximation and allows us to write down a corrected Schrödinger equation that encodes information about quantum-gravitational effects. The analytical dependence of the correction to the power spectrum on the wavenumber is obtained. Nonetheless, some numerical work is needed in order to obtain its precise value. Finally, it is shown that the correction turns out to be positive, which leads to an enhancement of the power spectrum especially prominent for large scales. We will also discuss whether this correction leads to a measurable effect in the cosmic microwave background anisotropies.
\end{abstract}

Keywords: quantum cosmology; slow-roll inflation; cosmic microwave background

\section{Introduction}

The lack of experimental guidance is still one of the main obstacles to advance in the construction and verification of the ultimate theory of quantum gravity [1]. There are several indications that the initial phases of the universe might be a good laboratory for such a purpose. In particular, during the inflationary phase of the primordial universe energies close to the Planck scale were present and the conditions for structure formation were set, which makes it plausible that Planck-scale physics might be directly related to the anisotropies of the cosmic microwave background (CMB) and, thus, be observable by our telescopes [2].

In this conference proceedings contribution based on our previous work published in [3,4], we review a computation of the quantum-gravity effects in the power spectra of both gauge-invariant scalar and tensor perturbations. For such a purpose, the perturbations of a homogeneous background universe with an inflaton field will be considered. The gauge-invariant master variables, which encode the complete physical information of the scalar and tensor perturbations, will be constructed in order to deal with the perturbative gauge invariance. The quantization of the whole system (background plus perturbations) will then be carried out following the canonical approach, which will lead to a master Wheeler-DeWitt equation. In order to solve it, a Born-Oppenheimer type of approximation will be applied by performing a power expansion in the inverse of the Planck mass. In this way, the search for a solution of the Wheeler-DeWitt equation will be translated into an infinite set of problems, one at every order of the expansion parameter. Finally, in order to keep the potential of the inflaton field as generic as possible, all the computations will be performed assuming a slow-roll regime. 


\section{Hamiltonian Framework for Classical Perturbations of an Inflationary Universe}

Perturbation theory is a very useful tool in many areas of physics and, in particular, in gravitational physics. The idea is fairly simple; one typically considers a known solution $g_{\mu v}$ of the Einstein equations as the background manifold. The perturbations of the metric $h_{\mu v}$ are then understood as fields propagating on that background, such that the dynamics of $h_{\mu v}$ is ruled by the linearized version of the Einstein equations.

In particular, the action of general relativity with a scalar matter field $\phi$ is given by,

$$
\mathcal{S}=\int \mathrm{d} t \int \mathrm{d}^{3} x\left(\pi^{i j} g_{i j, t}+\pi_{\phi} \phi, t-H\right),
$$

$\pi^{i j}$ and $\pi_{\phi}$ being the conjugate momentum of the spatial metric $g_{i j}$ and of the matter field, respectively. The Hamiltonian $H$ is a linear combination of constraints,

$$
H:=N \mathcal{H}\left(\pi_{\phi}, \phi, \pi^{i j}, g_{i j}\right)+N^{k} \mathcal{H}_{k}\left(\pi_{\phi}, \phi, \pi^{i j}, g_{i j}\right),
$$

with $N$ being the lapse function and $N^{k}$ the shift vector. While the first-order variation of the action $(\delta S=0)$ provides the background equations of motion, the second variation $\delta^{2} S$ provides an effective action functional for the linear perturbations:

$$
\frac{1}{2} \delta^{2} \mathcal{S}=\int \mathrm{d} x^{4}\left\{p^{i j} h_{i j, t}+\delta \pi_{\phi} \delta \phi_{, t}-\delta N \delta \mathcal{H}-\delta\left[N^{i}\right] \delta\left[\mathcal{H}_{i}\right]-\frac{N}{2} \delta^{2}[\mathcal{H}]-\frac{N^{i}}{2} \delta^{2}\left[\mathcal{H}_{i}\right]\right\} .
$$

In our approach, we intend to quantize all degrees of freedom, thus a complete action for the whole system is needed. This is obtained by considering the sum of both actions, which implies that the total Hamiltonian has the following form,

$$
H_{\text {total }}:=N\left(\mathcal{H}+\frac{1}{2} \delta^{2} \mathcal{H}\right)+N^{i}\left(\mathcal{H}_{i}+\frac{1}{2} \delta^{2} \mathcal{H}_{i}\right)+\delta N \delta \mathcal{H}+\delta N^{i} \delta \mathcal{H}_{i} .
$$

The equations of motion can be obtained by computing the Poisson brackets of different variables with this Hamiltonian or, equivalently, by taking variations of the full action. In particular, it is straightforward to see that this system has eight constraints:

$$
\begin{aligned}
& \left(\mathcal{H}+\frac{1}{2} \delta^{2} \mathcal{H}\right)=0, \quad\left(\mathcal{H}_{i}+\frac{1}{2} \delta^{2} \mathcal{H}_{i}\right)=0, \\
& \delta \mathcal{H}=0, \quad \delta \mathcal{H}_{i}=0 .
\end{aligned}
$$

The first line corresponds to the background constraints, corrected by terms quadratic in the perturbations, whereas the second line represents expressions linear in the perturbations and it corresponds to the perturbative gauge freedom. At this point, one could follow several routes in order to perform the quantization of this system. On the one hand, one could quantize the full system à la Dirac, without fixing any gauge classically. This approach was followed by Halliwell and Hawking (for a homogeneous and isotropic background) in a well-known paper [5]. On the other hand, one could either totally or partially fix the gauge classically and then proceed to the quantization. The problem of this approach is that when extracting physical results, it is difficult to know whether those results are gauge independent and thus physically meaningful.

In order to deal with the perturbative gauge freedom, there is an intermediate route which can be followed, which consists in constructing master gauge-invariant variables. Examples of such variables are Regge-Wheeler [6] and Zerilli [7] variables for the Schwarzschild background; and Mukhanov-Sasaki [8,9] variables for the Friedmann-Robertson-Walker universe. Technically, these variables are constructed by performing an adequate canonical transformation, with the requirement that in the new variables the perturbative constraints are trivialized. The great advantage 
of this approach is that one does not need to consider the quantization of the perturbative constraints (since they are trivial) and all the results obtained are automatically gauge invariant.

Let us now specialize all of the above theory to the Friedmann-Robertson-Walker universe. By using the conformal time $\eta$, the background metric is given as follows,

$$
\mathrm{d} s^{2}=a^{2}(\eta)\left(-\mathrm{d} \eta^{2}+\mathrm{dx}^{2}\right),
$$

with $a$ being the scale factor. Note that, in this homogeneous case, the shift vector is chosen to be vanishing, $N^{i}=0$, and thus the only constraint left from (4) will be the Hamiltonian constraint $\left(\mathcal{H}+\frac{1}{2} \delta^{2} \mathcal{H}\right)=0$.

As it is usually done, the perturbation tensor $h_{\mu \nu}$ is then decomposed into scalar, vector, and tensor components. The construction of master gauge-invariant quantities leads to three variables that contain the three physical degrees of freedom of the system (two corresponding to the gravitational wave and one to the scalar matter field): the Mukhanov-Sasaki variable $v$ encodes the physical information of the scalar sector, whereas the tensorial sector is by construction gauge invariant and the two polarizations of the gravitational wave will be named as $v^{(\lambda)}=a h^{(\lambda)}$, with $\lambda=+, \times$. The Hamiltonian constraint is then decomposed as follows,

$$
\left(\mathcal{H}+\frac{1}{2} \delta^{2} \mathcal{H}\right)=\mathcal{H}_{0}+\mathcal{H}_{\mathrm{S}}+\mathcal{H}_{\mathrm{T}}
$$

Here, $\mathcal{H}_{0}$ is the Hamiltonian of the background minisuperspace model,

$$
\mathcal{H}_{0}=\frac{\mathrm{e}^{-2 \alpha}}{2}\left[-\frac{\pi_{\alpha}^{2}}{m_{\mathrm{P}}^{2}}+\pi_{\phi}^{2}+2 \mathrm{e}^{6 \alpha} \mathcal{V}(\phi)\right]
$$

where $\pi_{\alpha}$ is the conjugate momentum of $\alpha:=\ln a, \mathcal{V}(\phi)$ is the potential of the inflaton field, and we have defined the reduced Planck mass $m_{\mathrm{P}}^{2}:=\frac{3}{4 \pi G}$. Furthermore, $\mathcal{H}_{\mathrm{S}}$ and $\mathcal{H}_{\mathrm{T}}$ are the Hamiltonians corresponding to the scalar and tensorial sector, respectively. These two are Hamiltonians of parametric harmonic oscillators. In particular, the scalar one can be written as follows,

$$
\int \mathrm{d}^{3} x \mathcal{H}_{\mathrm{S}}=\frac{1}{2} \int \mathrm{d}^{3} \mathbf{k}\left(\pi_{\mathbf{k}} \bar{\pi}_{\mathbf{k}}+\omega_{\mathrm{S}}^{2}(\eta) v_{\mathbf{k}} \bar{v}_{\mathbf{k}}\right),
$$

where $\pi_{\mathbf{k}}$ is the conjugate momentum of the Mukhanov-Sasaki variable $v_{k}$, and the frequency is defined as $\omega_{S}^{2}(\eta)=k^{2}-\frac{z^{\prime \prime}}{z}, z$ being a given function of background objects. The Hamiltonian of the tensor part has exactly the same form, the only difference being that the frequency is given by $\omega_{\mathrm{T}}^{2}(\eta)=k^{2}-\frac{a^{\prime \prime}}{a}$.

\section{Quantization and Semiclassical Approximation}

The quantization of the Hamiltonian constraint (7) gives rise to a master Wheeler-DeWitt equation. By imposing an adequate product ansatz for the full wave function, and assuming that different $\mathbf{k}$-modes are independent (which translates to assuming that the action of the Hamiltonian for every $\mathbf{k}$ defined below in (12) is negligible when acting on another $\mathbf{k}^{\prime}$ ), one obtains the following equation for every mode $\mathbf{k}$ as well as for both the scalar and tensor sectors:

$$
\frac{1}{2}\left\{\mathrm{e}^{-2 \alpha}\left[\frac{1}{m_{\mathrm{P}}^{2}} \frac{\partial^{2}}{\partial \alpha^{2}}-\frac{\partial^{2}}{\partial \phi^{2}}+2 \mathrm{e}^{6 \alpha} \mathcal{V}(\phi)\right]-\frac{\partial^{2}}{\partial v_{\mathbf{k}}^{2}}+\omega^{2}(\eta) v_{\mathbf{k}}^{2}\right\} \Psi_{\mathbf{k}}\left(\alpha, \phi, v_{\mathbf{k}}\right)=0 .
$$

Note that in this equation the minisuperspace variables and the part involving inhomogeneous degrees of freedom for a given $\mathbf{k}$ appear on the same footing, and the fiducial volume of the minisuperspace has been absorbed in the minisuperspace variables. The frequency $\omega$ should be 
taken as $\omega_{\mathrm{S}}$ or $\omega_{\mathrm{T}}$, depending on whether we are dealing with the scalar or tensorial sector respectively. To simplify the presentation from here on, if not otherwise stated, we will only consider the scalar sector, which is more involved, and thus $\omega$ should be understood as $\omega_{S}$; in the last Section 6 , we will also summarize the results corresponding to the tensorial part.

Following the approach presented in [10,11], and also used in $[12,13]$, the next step of the analysis involves a power expansion in the inverse of the squared Planck mass $m_{\mathrm{P}}^{2}$. At leading order, one recovers the Hamilton-Jacobi equation, which encodes the classical dynamics of the system. At the next order, the approximation of quantum field theory on a curved (classical) spacetime is obtained via the following Schrödinger equation:

$$
\mathrm{i} \frac{\partial}{\partial \eta} \psi_{\mathbf{k}}^{(0)}=\mathcal{H}_{\mathbf{k}} \psi_{\mathbf{k}}^{(0)}
$$

where the matter Hamiltonian operator is, as usual, the one corresponding to a parametric oscillator,

$$
\mathcal{H}_{\mathbf{k}}:=-\frac{1}{2} \frac{\partial^{2}}{\partial v_{\mathbf{k}}^{2}}+\frac{1}{2} \omega^{2}(\eta) v_{\mathbf{k}}^{2} .
$$

At the following order, new physics is obtained and the equation to be solved is a modified Schrödinger equation:

$$
\mathrm{i} \frac{\partial}{\partial \eta} \psi_{\mathbf{k}}^{(1)}=\mathcal{H}_{\mathbf{k}} \psi_{\mathbf{k}}^{(1)}-\frac{\psi_{\mathbf{k}}^{(1)}}{2 m_{\mathrm{P}}^{2} \psi_{\mathbf{k}}^{(0)}}\left[\frac{\left(\mathcal{H}_{\mathbf{k}}\right)^{2}}{V} \psi_{\mathbf{k}}^{(0)}+\mathrm{i} \frac{\partial}{\partial \eta}\left(\frac{\mathcal{H}_{\mathbf{k}}}{V}\right) \psi_{\mathbf{k}}^{(0)}\right],
$$

where an auxiliary potential has been defined as

$$
V(a, \phi):=\frac{2 a^{4}}{m_{\mathrm{P}}^{2}} \mathcal{V}(\phi)
$$

Note that the quantum-gravitational effects are encoded in the last term of Equation (13). In fact, this term is just a product between $\psi_{\mathbf{k}}^{(1)}$ and another term that depends on background quantities and the wave function $\psi_{\mathbf{k}}^{(0)}$. This latter is in general a complex function and thus this correction term is a possible source of unitarity violation. In order to avoid such an undesirable effect and to construct a consistent framework, we will just consider the real part of this term. The appearance of these unitarity-violating terms has been discussed in detail in our previous work $[3,4]$ and will be subject to further investigations.

\section{Gaussian Ansatz and Power Spectra}

In summary, we need to solve Equations (11) and (13) for wave functions $\psi_{\mathbf{k}}^{(0)}$ and $\psi_{\mathbf{k}}^{(1)}$. Since the matter Hamiltonian (12) corresponds to a parametric oscillator, it is thus natural to consider a Gaussian ansatz as follows,

$$
\psi_{\mathbf{k}}\left(\eta, v_{\mathbf{k}}\right)=N_{\mathbf{k}}(\eta) \mathrm{e}^{-\frac{1}{2} \Omega_{\mathbf{k}}(\eta) v_{\mathbf{k}}^{2}}
$$

for both $\psi_{\mathbf{k}}^{(0)}$ and $\psi_{\mathbf{k}}^{(1)}$. Here, and in the following, the superscript $(0)$ stands for the uncorrected and (1) for the corrected case. Assuming this form for the wave functions, the uncorrected and corrected power spectra are directly given by the real part of the corresponding Gaussian width, $\Omega_{\mathbf{k}}^{(0)}$ and $\Omega_{\mathbf{k}}^{(1)}$, respectively. More precisely, for the scalar perturbations the corresponding power spectrum is given by

$$
\mathcal{P}_{\mathrm{S}}^{(1)}(k)=\frac{4 \pi G}{a^{2} \epsilon} \frac{k^{3}}{2 \pi^{2}} \frac{1}{2 \Re_{\mathfrak{e}} \Omega_{\mathrm{k}}^{(1)}} \approx \mathcal{P}_{\mathrm{S}}^{(0)}(k)\left\{1+\Delta_{\mathrm{S}}\right\}
$$


where $\epsilon$ is the slow-roll parameter, which will be defined in (21) below. Furthermore, $\mathcal{P}_{\mathrm{S}}^{(0)}(k)$ is the usual spectrum that is obtained by quantum field theory methods [14]. In this setting, this would be obtained by replacing $\Omega_{\mathbf{k}}^{(1)}$ by $\Omega_{\mathbf{k}}^{(0)}$ in this latter equation. Therefore, the relative difference between the two (corrected and uncorrected) spectra is encoded in the object

$$
\Delta_{\mathrm{S}}:=\lim _{\eta \rightarrow 0} \frac{\Re_{\mathfrak{e}} \Omega_{\mathbf{k}}^{(0)}-\Re_{\mathfrak{e}} \Omega_{\mathbf{k}}^{(1)}}{\Re_{\mathfrak{e}} \Omega_{\mathbf{k}}^{(0)}},
$$

evaluated in the large-scale limit.

Since the quantities of interest are the Gaussian widths, let us present the equations obeyed by them in both the uncorrected and the corrected cases:

$$
\begin{aligned}
& \mathrm{i} \Omega_{\mathbf{k}}^{(0) \prime}(\eta)=\left(\Omega_{\mathbf{k}}^{(0)}(\eta)\right)^{2}-\omega^{2}(\eta), \\
& \mathrm{i} \Omega_{\mathbf{k}}^{(1) \prime}(\eta)=\left(\Omega_{\mathbf{k}}^{(1)}(\eta)\right)^{2}-\widetilde{\omega}^{2}(\eta),
\end{aligned}
$$

where the prime denotes the derivative with respect to the conformal time. As can be seen, the equations are exactly the same, with the only exception that for the former case the frequencies are the ones defined after Equation (9), $\omega_{\mathrm{S}}$ or $\omega_{\mathrm{T}}$, depending on whether one is considering the scalar or tensorial sector, whereas for the latter case the corrected frequencies $\widetilde{\omega}$ are defined as follows:

$$
\widetilde{\omega}^{2}:=\omega^{2}-\frac{1}{2 m_{\mathrm{P}}^{2} V} \Re \mathfrak{e}\left[\left(3 \Omega_{\mathbf{k}}^{(0)}-\mathrm{i}(\ln V)^{\prime}\right)\left(\omega^{2}-\left(\Omega_{\mathbf{k}}^{(0)}\right)^{2}\right)+2 \mathrm{i} \omega \omega^{\prime}\right] .
$$

In summary, in order to get the quantum-gravity correction to the power spectrum, one needs to solve Equations (18) and (19) and compute then $\Delta_{\mathrm{S}}$ as defined in (17). Let us briefly comment about the initial conditions to be considered. The usual initial conditions that are considered in the quantum-field-theoretical approach are given by the so-called Bunch-Davies vacuum, which implies that at the beginning of inflation, when the mode is well inside the horizon, it behaves as a mode on a Minkowski background, without feeling the curvature. In this setting, this implies to choose $\Omega_{\mathbf{k}}^{(0)}=\omega$ at the onset of inflation. For the corrected case, we mimic this initial data and also request that the mode is oscillating with constant frequency and amplitude, which is translated to the initial condition $\Omega_{\mathrm{k}}^{(1)}=\widetilde{\omega}$. The difference with the uncorrected case is that the frequency of the mode, even if constant at the beginning, gets modified.

\section{Slow-Roll Approximation}

The numerical value of $\Delta_{S}$ will depend on the inflaton potential $\mathcal{V}(\phi)$. Instead of fixing this potential, we will consider the slow-roll regime, which is quite generic and includes several types of potentials. Therefore, we define the two slow-roll parameters,

$$
\epsilon:=-\frac{\dot{H}}{H^{2}}, \quad \delta:=\epsilon-\frac{\dot{\epsilon}}{2 H \epsilon},
$$

and their combination $\gamma:=2 \epsilon-\delta$. In these definitions, $H$ is the Hubble rate and the dot stands for the derivative with respect to cosmic time.

By performing an expansion in these parameters, and dropping quadratic and higher-order terms, one can obtain the following form for the scalar and tensorial frequencies [14]:

$$
\omega_{\mathrm{S}}^{2}=k^{2}-\frac{2+3 \gamma}{\eta^{2}}, \quad \omega_{\mathrm{T}}^{2}=k^{2}-\frac{2+3 \epsilon}{\eta^{2}} .
$$


Note that $\gamma=\epsilon$ converts the scalar equation into the tensorial one. We will use this fact in the following section to recover the tensorial results from the ones of the scalar sector.

In addition, at this level of approximation, the auxiliary potential $V$ (14) can be written in the following way [4]:

$$
V(\eta)=\frac{1}{H_{k}^{2} \eta^{4}(k \eta)^{2 \epsilon}}\left(1+\frac{11 \epsilon}{3}\right)
$$

where $H_{k}$ is the value of the Hubble factor at the horizon exit, which depends on the mode $k$. With these expressions at hand, one can get the form of the corrected frequencies (20) in the slow-roll approximation and thus write our main Equations (18) and (19) in this regime without any reference to the potential of the inflaton field.

\section{Results}

The main Equation (19) is quite complicated and one needs to resort to numerical methods in order to solve it in the slow-roll regime. Nevertheless, it is possible to obtain the analytical dependence of the corrections to the power spectrum with numerical coefficients. More precisely, for the scalar sector it can be written as follows,

$$
\Delta_{\mathrm{S}} \simeq \frac{H_{k}^{2}}{m_{\mathrm{P}}^{2}}\left(\frac{\bar{k}}{k}\right)^{3}[0.988+3.14 \epsilon-2.56 \delta] .
$$

We had to introduce a reference scale $\bar{k}$ at this point, for which the most reasonable choice in order to give an estimate of the corrections seems to be the pivot scale used in the Planck data analysis. The issue in choosing the most appropriate reference scale is, however, still under debate and was also discussed in [15].

The most important feature of this correction is that it is not scale independent, since it goes as $k^{-3}$, and it is thus more relevant for large scales. This is something expected since modes with small wave number are the first to exit the Hubble horizon and therefore experience stronger quantum-gravity effects. Furthermore, since the absolute value of $\epsilon$ and $\delta$ is smaller than $10^{-2}$, the correction term (24) leads to an enhancement of the power spectrum. One can also analytically compute the corrections for the spectral index $n_{\mathrm{S}}$ and its running. In particular, the spectral index is corrected by a negative term, whereas its running gets a positive correction [4]. All these corrections are of the order of $\Delta_{\mathrm{S}}$.

Concerning the corrections to the tensorial power spectrum, as commented in the previous section, these can be obtained from the result of the scalar part, just by imposing $\gamma=\epsilon$. In this way, one obtains

$$
\Delta_{\mathrm{T}} \simeq \frac{H_{k}^{2}}{m_{\mathrm{P}}^{2}}\left(\frac{\bar{k}}{k}\right)^{3}[0.988+0.58 \epsilon]
$$

The form of this correction is qualitatively the same as the scalar one. Therefore, the main features and conclusions drawn above for the scalar sector are also valid for the tensorial case. We can also compute the correction to the tensor-to-scalar ratio $r$, which leads to

$$
r^{(1)}:=\frac{\mathcal{P}_{\mathrm{T}}^{(1)}(k)}{\mathcal{P}_{\mathrm{S}}^{(1)}(k)} \approx 16 \epsilon\left(1+2.56 \frac{H_{k}^{2}}{m_{\mathrm{P}}^{2}}\left(\frac{\bar{k}}{\bar{k}}\right)^{3}(\delta-\epsilon)\right)
$$

Regarding the possible observability of these effects, taking into account data of the Planck mission [16], it is possible to give an estimate of the correction terms $\Delta_{\mathrm{S}}$ and $\Delta_{\mathrm{T}}$. Given that the tensor-to-scalar ratio deduced from the Planck data is less than $r \lesssim 0.11$, the ratio $H_{\text {inf }}^{2} / m_{\mathrm{P}}^{2}$, where $H_{\text {inf }}$ is the Hubble rate during inflation, is bounded to be less than 


$$
\frac{H_{\mathrm{inf}}^{2}}{m_{\mathrm{P}}^{2}} \sim \frac{2 r}{0.01}\left(\frac{10^{16} \mathrm{GeV}}{m_{\mathrm{P}}}\right)^{4} \lesssim 1.7 \times 10^{-10} .
$$

This leads to the following upper bound for our quantum-gravitational correction terms

$$
\left|\Delta_{\mathrm{S}}\right| \approx\left|\Delta_{\mathrm{T}}\right| \lesssim 2 \times 10^{-10}
$$

where the ratio between $\Delta_{\mathrm{S}}$ and $\Delta_{\mathrm{T}}$ is around $\Delta_{\mathrm{S}} / \Delta_{\mathrm{T}} \approx 1.02$. For the bound on the correction $\Delta r:=r^{(1)}-r^{(0)}$ of the tensor-to-scalar ratio, we obtain

$$
\frac{\Delta r}{r^{(0)}} \approx-4 \times 10^{-12}
$$

In order to see whether these bounds lead to corrections that are too small to be observed with the current experimental data, we calculate the quantities $C_{\ell}$ that are used to describe the CMB anisotropies. They are defined as

$$
C_{\ell}^{(i)}=\int_{0}^{\infty} \frac{\mathrm{d} k}{3 k} \mathcal{P}_{\mathrm{S}}^{(i)}(k) j_{\ell}^{2}\left(k\left[\eta_{\text {hor }}-\eta_{\mathrm{rec}}\right]\right),
$$

where we introduced the conformal time at horizon crossing $\eta_{\text {hor }}$ and recombination $\eta_{\text {rec }}$ and used $i=0,1$ to indicate the uncorrected or corrected quantities, respectively. We can, in fact, calculate the correction to the $C_{\ell}$ expressed as $\Delta C_{\ell}:=C_{\ell}^{(1)}-C_{\ell}^{(0)}$ analytically and obtain

$$
\begin{aligned}
\Delta C_{\ell} & \approx \frac{1}{4 \pi^{2}} \int_{0}^{\infty} \frac{\mathrm{d} k}{k \epsilon}\left(\frac{H_{k}}{m_{\mathrm{P}}}\right)^{4}\left(\frac{\bar{k}}{\bar{k}}\right)^{3} j_{\ell}^{2}\left(k\left[\eta_{\mathrm{hor}}-\eta_{\mathrm{rec}}\right]\right) \\
& \approx \frac{3}{4 \pi \epsilon}\left(\frac{H_{k}}{m_{\mathrm{P}}}\right)^{4} \frac{\left|\bar{k}\left(\eta_{\mathrm{hor}}-\eta_{\mathrm{rec}}\right)\right|^{3}}{(2 \ell-3)(2 \ell-1)(2 \ell+1)(2 \ell+3)(2 \ell+5)} .
\end{aligned}
$$

For $\ell=2$, where the correction is most prominent, we thus get

$$
\frac{\Delta C_{2}}{C_{2}^{(0)}} \approx 0.12\left(\frac{H_{k}}{m_{\mathrm{P}}}\right)^{2}\left|\bar{k}\left(\eta_{\mathrm{hor}}-\eta_{\mathrm{rec}}\right)\right|^{3} .
$$

This allows us to compare this correction to cosmic variance, which fundamentally restricts the accuracy with which we can measure the $\mathrm{CMB}$ anisotropies. The statistical uncertainty arising from cosmic variance can be expressed by

$$
\frac{\Delta C_{\ell}^{\mathrm{CV}}}{C_{\ell}^{(0)}}=\sqrt{\frac{2}{2 \ell+1}},
$$

such that for $\ell=2$, we have $\Delta C_{2}^{\mathrm{CV}} / C_{2}^{(0)} \approx 0.63$. Since we can estimate that $\left|\bar{k}\left(\eta_{\text {hor }}-\eta_{\text {rec }}\right)\right|^{3} \approx$ $5 \times 10^{4}$ [4], the order of magnitude that the ratio $H_{k}^{2} / m_{\mathrm{P}}^{2}$ would need to have, such that the quantum-gravitational corrections obtained here overcome cosmic variance and could thus be measurable, can be estimated to be

$$
\frac{H_{k}^{2}}{m_{\mathrm{P}}^{2}} \gtrsim 10^{-4},
$$

which is way above the bound obtained in (27). Hence, we need to conclude that these corrections are unfortunately too small to be observed with the current CMB data. Nevertheless, our calculations show that concrete predictions can be obtained from approaches to quantum gravity. The question as to whether there are other situations where such effects can be observed, remains presently open. 
Acknowledgments: We thank Claus Kiefer for collaboration on the subject under discussion in this proceedings contribution. D. B. is supported by project FIS2014-57956-P of the Spanish Ministry of Economy and Competitiveness and by project IT956-16 of the Basque Government. The research of M. K. was financed by the Polish National Science Center Grant DEC-2012/06/A/ST2/00395.

Author Contributions: The authors contributed equally to this work.

Conflicts of Interest: The authors declare no conflict of interest.

\section{References}

1. Kiefer, C. Quantum Gravity, 3rd ed.; International Series of Monographs on Physics 155; Oxford University Press: Oxford, UK, 2012.

2. Kiefer, C.; Krämer, M. Can effects of quantum gravity be observed in the cosmic microwave background? Int. J. Mod. Phys. D 2012, 21, 1241001.

3. Brizuela, D.; Kiefer, C.; Krämer, M. Quantum-gravitational effects on gauge-invariant scalar and tensor perturbations during inflation: The de Sitter case. Phys. Rev. D 2016, 93, 104035.

4. Brizuela, D.; Kiefer, C.; Krämer, M. Quantum-gravitational effects on gauge-invariant scalar and tensor perturbations during inflation: The slow-roll approximation. Phys. Rev. D 2016, 94, 123527.

5. Halliwell, J.J.; Hawking, S.W. Origin of structure in the universe. Phys. Rev. D 1985, 31, 1777.

6. Regge, T.; Wheeler, J.A. Stability of a Schwarzschild singularity. Phys. Rev. 1957, 108, 1063.

7. Zerilli, F.J. Effective potential for even-parity Regge-Wheeler gravitational perturbation equations. Phys. Rev. Lett. 1970, 24, 737.

8. Kodama, H.; Sasaki, M. Cosmological perturbation theory. Prog. Theor. Phys. 1984, 78, 1-166.

9. Mukhanov, V.F. Quantum theory of gauge-invariant cosmological perturbations. Zh. Eksp. Teor. Fiz. 1988, $94,1$.

10. Kiefer, C.; Singh, T.P. Quantum gravitational corrections to the functional Schrödinger equation. Phys. Rev. D 1991, 44, 1067.

11. Kiefer, C. The semiclassical approximation to quantum gravity. Lect. Notes Phys. 1994, 434, 170.

12. Kiefer, C.; Krämer, M. Quantum gravitational contributions to the cosmic microwave background anisotropy spectrum. Phys. Rev. Lett. 2012, 108, 021301.

13. Bini, D.; Esposito, G.; Kiefer, C.; Krämer, M.; Pessina, F. On the modification of the cosmic microwave background anisotropy spectrum from canonical quantum gravity. Phys. Rev. D 2013, 87, 104008.

14. Peter, P.; Uzan, J.-P. Primordial Cosmology; Oxford University Press: Oxford, UK, 2009.

15. Kamenshchik, A.Y.; Tronconi, A.; Venturi, G. Quantum cosmology and the evolution of inflationary spectra. Phys. Rev. D 2016, 94, 123524.

16. Ade, P.A.; Aghanim, N.; Arnaud, M.; Ashdown, M.; Aumont, J.; Baccigalupi, C.; Banday; A.J.; Barreiro, R.B.; Barreiro, J.G.; Battaner, E.; et al; Planck 2015 results. XIII. Cosmological parameters. Astron. Astrophys. 2016, 594, A13.

(C) 2018 by the authors. Licensee MDPI, Basel, Switzerland. This article is an open access article distributed under the terms and conditions of the Creative Commons Attribution (CC BY) license (http:/ / creativecommons.org/licenses/by/4.0/). 